\title{
Tiaochang Xiaoyan extract tablets ameliorate chronic inflammation by activating macrophage lysosomes in chronic colitis rats
}

\author{
Shiying Wang ${ }^{1,2} \wedge$, Chun Guo ${ }^{1}$, Tao Zhang ${ }^{3}$, Cailing Zhong ${ }^{1}$, Xiying Zhao ${ }^{1}$, Yisheng Su${ }^{1}$, Wei Wei ${ }^{3}$, \\ Beiping Zhang $^{1 \wedge}$
}

${ }^{1}$ Department of Gastroenterology, The Second Affiliated Hospital of Guangzhou University of Chinese Medicine, Guangzhou, China; ${ }^{2}$ Guangdong Province Engineering Technology Research Institute of Traditional Chinese Medicine, Guangzhou, China; ${ }^{3}$ Department of Gastroenterology, Beijing Key Laboratory of Functional Gastrointestinal Disorders Diagnosis and Treatment of Traditional Chinese Medicine, Wangjing Hospital, China Academy of Chinese Medical Sciences, Beijing, China

Contributions: (I) Conception and design: B Zhang, S Wang; (II) Administrative support: B Zhang, W Wei; (III) Provision of study materials: S Wang, C Guo, T Zhang, C Zhong, X Zhao, Y Su; (IV) Collection and assembly of data: S Wang, C Guo; (V) Data analysis and interpretation: T Zhang, C Zhong, X Zhao, Y Su; (VI) Manuscript writing: All authors; (VII) Final approval of manuscript: All authors.

Correspondence to: Wei Wei. Department of Gastroenterology, Beijing Key Laboratory of Functional Gastrointestinal Disorders Diagnosis and Treatment of Traditional Chinese Medicine, Wangjing Hospital, China Academy of Chinese Medical Sciences, No. 6, Wangjing Middle Ring South Road, Chaoyang District, Beijing 100102, China. Email: sxxtyy@sina.com; Beiping Zhang. Department of Gastroenterology, The Second Affiliated Hospital of Guangzhou University of Chinese Medicine, 111 Dade Road, Yuexiu District, Guangzhou 510120, China. Email: doctorzbp@163.com.

Background Tiaochang Xiaoyan tablet (TCXYT) is a traditional Chinese medicine prescription derived from
the Xianglian pill, which is a traditional Chinese medicine for treating chronic dysentery recorded in the
Taiping Huimin Heji Bureau [1078-1085]. For many years, TCXYT has been used to treat ulcerative colitis,
however, its therapeutic mechanism is still unclear. In the present study, we used colonic lamina propria
macrophages (LPM) and mouse-derived macrophage cell line RAW264.7 cells as the research objects, with
the aim of exploring the therapeutic effects and mechanisms of TCXYT on colitis. Methods: We used 2,4,6-trinitrobenzenesulfonic acid (TNBS) to induce a rat model of chronic colitis, and normal rats as the control. The disease activity index (DAI) and colonic histopathological changes of rats were used to evaluate the severity of colitis. Rats were divided into the control group; model group; high, middle-, and low-dose TCXYT group; and the hydroxychloroquine sulfate group. TCXYT was administered by gavage on the $3 \mathrm{rd}$ day after model replication and lasted for 7 days. The doses used for the high-, middle-, and low-dose TCXYT groups were 0.8, 0.4 and $0.2 \mathrm{~g} / \mathrm{kg}$, respectively. Enzyme-linked immunosorbent assay was used to detect the serum concentration of cytokines. Western blot was used to detect the expressions of Toll-like receptor 9 (TLR9), myeloid differentiation primary response 88 (MyD88), interleukin (IL) receptor-associated kinase (IRAK) 1, and IRAK4 in colonic LPM and RAW264.7 cells. Immunofluorescence was used to detect lysosomal activity. The chemical constituents of TCXYT were separated and identified based on Q-Orbitrap high resolution LC/MS data.

Results: TCXYT promoted the repair of colonic mucosal injury, attenuated inflammation, increased lysosome activity in macrophages, and decreased the DAI in rats with colitis compared with those in the model group. TCXYT decreased the serum concentrations of IL-1 $\beta$ and tumor necrosis factor- $\alpha$ (TNF- $\alpha$ ), increased those of IL-4 and IL-10, and decreased the TLR9, MyD88, IRAK1, and IRAK4 protein levels in LPM and RAW264.7 cells compared to the model group.

Conclusions: TCXYT could ameliorate colon inflammation and CD11 ${ }^{+}$macrophage infiltration in rats with chronic colitis. This effect may be mediated by activating lysosomes in macrophages by inhibiting the TLR9/MyD88/IRAK signaling pathway.

^ ORCID: Shiying Wang, 0000-0002-1005-0381; Beiping Zhang, 0000-0003-2979-9226. 


\section{Keywords: Tiaochang Xiaoyan tablet (TCXYT); ulcerative colitis; macrophages; lysosome; inflammation}

Submitted Jan 13, 2021. Accepted for publication Feb 04, 2021.

doi: $10.21037 /$ apm-21-250

View this article at: http://dx.doi.org/10.21037/apm-21-250

\section{Introduction}

Ulcerative colitis (UC) is a chronic and progressive colonic inflammatory disease with recurrent mucosal inflammation and mucosal damage. Recurrent inflammation in colonic mucosa is the most important pathological factor that leads to the occurrence of mucosal ulcers $(1,2)$, and has been linked to increased risk of ulcer-associated colorectal cancer (3). It was found in previous studies that the occurrence of colonic mucosal inflammation in UC patients is closely related to mucosal hypoxia, abnormal activation of inflammatory cells, and autophagy disorder $(4,5)$. Abnormal colonic mucosal inflammation is not only related to the toxin and pathogen translocation to submucosa that is caused by the increased permeability of UC colonic mucosa but is also closely related to the clearance of apoptotic body and necrotic fragments and abnormal immune mediators, and then destroy the immune homeostasis $(6,7)$. Some studies have suggested that the accumulation of apoptotic fragments can lead to tissue damage and the destruction of immune homeostasis, which further leads to abnormal autoimmune and immune homeostasis $(8,9)$. In addition, it was discovered that mucosal damage in colonic mucosa of UC is related to abnormal immune homeostasis $(7,10)$, which is caused by the formation of an abnormal immune complex in colon mucosa. In this process, phagocytosis of macrophages plays an important role in removing pathogenic microorganisms, the clearance of apoptotic body, and other abnormal immune mediators, and maintaining the immune homeostasis of colonic mucosa (9). The lysosome is considered an important organelle in macrophages, which plays an important role in regulating the immune functions of macrophages. Previous studies have found that the maturation of macrophage lysosomes is important for the stabilization of macrophage-mediated autophagy, immune presentation, and other functions $(11,12)$. However, the mechanism of lysosomal function in macrophages is unclear. The Tolllike receptor (TLR) signaling pathway plays an important role in the development of colonic mucosal inflammation in UC (13). Previous studies have found that the activation of the TLR9 signaling pathway in colonic lamina propria macrophages (LPM) can significantly inhibit macrophage autophagy, aggravate the inflammatory response, and increase the apoptosis of mucosal epithelial cells (13-16). Furthermore, the dysfunction of lysosomal activity in LPM has been found to be the main cause of macrophage dysfunction (14). In addition, the TLR9 signaling pathway is involved in the regulation of autophagy of LPM in UC (13), but the effect of the TLR9 signaling pathway on the regulation of lysosomal structure and the function of LPM is unclear. Therefore, the aim of the present study was to explore the role and mechanism of lysosomes in LPM on chronic inflammation.

UC belongs to the categories of diarrhea, dysentery, and intestinal wind of traditional Chinese medicine. Chronic recurrence belongs to the categories of rest dysentery and chronic dysentery of traditional Chinese medicine, which are characterized by long unhealing and easy recurrence. According to the theory of traditional Chinese medicine, it is generally believed that ulcerative colitis is mainly caused by spleen and kidney deficiencies and stagnation of dampness, heat, phlegm, and qi (17). The onset of UC is believed to be related to pathogenic toxins in the body (18). It is believed that the pathogenic toxins are the root of UC, which can cause UC. Therefore, in terms of treatment, detoxification is considered the main method of treatment. Detoxification is used throughout the whole process of $\mathrm{UC}$ and is supplemented by heat and damp clearance, qi activation, and blood circulation. TCXYT is derived from the Xianglian pill, which is a traditional Chinese medicine for treating chronic dysentery recorded in the Taiping Huimin Heji Bureau [1078-1085]. TCXYT consists of Radix Astragali, Lindera aggregata, Rbizoma coptidis, Oldenlandia diffusa and coix seed. Our previous research found that TCXYT can effectively inhibit the chronic inflammation of colonic mucosa and promote the repair of colonic ulcers in patients with ulcerative colitis; the mechanism may be related to inhibiting the autophagy function of colon cells by regulating the balance of pro-inflammatory and antiinflammatory factors (19-21). Our findings indicated that TCXYT can regulate the lysosomal activity of macrophages 
in the colon of rats with chronic colitis induced by 2,4,6-trinitrobenzenesulfonic acid (TNBS). We conducted the following research.

We present the following article in accordance with the ARRIVE reporting checklist (available at http://dx.doi. org/10.21037/apm-21-250).

\section{Methods}

\section{Animals}

Sprague-Dawley rats $(140-160 \mathrm{~g}$, certificate No. 4400210019232) were obtained from the Experimental Animal Center of Southern Medical University, Guangzhou, China (license No. scxk-Guangdong-2006-0015). The Institutional Animal Care and Use Committee of The Second Affiliated Hospital of Guangzhou University of Chinese Medicine approved all of the procedures involving the rats (animal ethics approval No. 2016021-2). Experiments were performed in compliance with the Institutional Animal Care and Use Committee of Guangzhou University of Chinese Medicine's guidelines for the care and use of animals. We quantitatively analyzed the weight, fecal texture, and other metrics of the rats. The rats were housed in a pathogen-free environment and were allowed to acclimatize for 7 days before use. These rats were divided into the control group $(\mathrm{n}=6)$; model group; the high-, middle-, and low-dose TCXYT groups; and the hydroxychloroquine sulfate (HS) group. (The dosage of TCXYT in the trial is calculated based on the clinical dosage. For example, the dosage for rats is $60 \mathrm{mg} / \mathrm{kg} \times$ $70 \mathrm{~kg} \times 0.018 / 200 \mathrm{~g}=378 \mathrm{mg} / \mathrm{kg}$. In the experiment, each rat weighed about $400 \mathrm{~g}$, and given $2.5 \mathrm{~mL}$ of the drug solution with a concentration of $0.06 \mathrm{~g} / \mathrm{mL}$ by gavage. Therefore, the dosage of each rat was $375 \mathrm{mg} / \mathrm{kg}$. In order to facilitate the configuration of the drug, $0.4 \mathrm{~g} / \mathrm{kg}$ of the trial drug was used as the medium dose, $1 / 2$ times was the low dose, and 2 times was the high dose.)

\section{Reagents}

TNBS and lipopolysaccharide (LPS) were purchased from Sigma (St Louis, MO, USA). The Lysosome Staining Kit was purchased from Abnova (Taipei City, Taiwan, China). Enzyme-linked immunosorbent assay (ELISA) kits for interleukin (IL)-1 $\beta$, IL-4, IL-10, and tumor necrosis factor- $\alpha(\mathrm{TNF}-\alpha)$ were from R\&D Systems (Minneapolis, MN, USA). HS (an inhibitor of autophagy and TLR7/9) was obtained from Selleck Chemicals (Houston, TX, USA). Mouse interferon- $\gamma$ (IFN- $\gamma$ ), IL-1R-associated kinase (IRAK) 1, and IRAK4 were from Cell Signaling Technology (Danvers, MA, USA). CD11c (ab11029), Microtubule Associated Protein 1 Light Chain 3 Beta (LC3B, ab192890), TLR9 (ab134368), and myeloid differentiation primary response 88 (MyD88) antibodies, goat anti-rabbit antibodies, and rabbit anti-mouse antibodies were from Abcam (Cambridge, UK).

\section{Drugs}

TCXYT is a herbal preparation that consists of Radix Astragali seu Hedysari, Radix Linderae, Rbizoma Coptidis, Herba Hedyotis and Semen Coicis (Table S1). In addition, all herbal medicines were purchased from Lingnan Traditional Chinese Medicine Co. Ltd. (Guangzhou, China) and provided by the Department of Pharmacy at The Second Affiliated Hospital of Guangzhou University of Chinese Medicine. Quality of herbal medicines was tested according to the standards of the Pharmacopoeia of the People's Republic of China [2015] before the experiment.

\section{Sample preparation and Q-Orbitrap bigh resolution LC/ MS analysis}

The methods and further details used have been described elsewhere $(22,23)$. The extract of TCXYT was powdered and passed through 100-mesh sieves. An aliquot of $50 \mathrm{mg}$ of powder was extracted in $10 \mathrm{~mL}$ of $70 \%$ methanol (v/v) for $30 \mathrm{~min}$ by ultrasonication $(40 \mathrm{kHz}, 300 \mathrm{~W})$. The sample was maintained at room temperature for $5 \mathrm{~min}$, and the supernatant was filtered through a $0.22-\mu \mathrm{m}$ membrane before use. An aliquot of $1 \mu \mathrm{L}$ was injected for analysis.

A UHPLC Ultimate 3000 instrument coupled with a Q-Exactive Hybrid Quadrupole-Orbitrap mass spectrometer (Thermo Fisher Scientific, Waltham, MA, USA) was used. Samples were separated on a Welch Ultimate Polar reversed-phase C18 column $(150 \times 2.1 \mathrm{~mm} 1.8 \mu \mathrm{m})$. Mobile phase $\mathrm{A}$ is an aqueous solution containing $5 \%$ methanol- $0.1 \%$ formic acid and $2 \mathrm{mmol} / \mathrm{L}$ ammonium formate; and mobile phase B was a methanol solution containing $15 \%$ isopropanol $+0.1 \%$ formic acid. A gradient elution program was used as follows: $0-5 \mathrm{~min}, 20 \% \mathrm{~A}$; $5-10 \mathrm{~min}, 20-50 \% \mathrm{~A} ; 10-15 \mathrm{~min}$, $50-80 \% \mathrm{~A}$; and $15-25 \mathrm{~min}, 80-95 \% \mathrm{~A}$. The flow rate was $0.30 \mathrm{~mL} / \mathrm{min}$, and the column temperature was maintained at $35^{\circ} \mathrm{C}$. The mass spectrometer was operated in the 
(+/-) electrospray ionization (ESI) mode. The parameters were as follows: spray voltage: $3.8 \mathrm{kV}$, sheath gas pressure: 40 arb, Aux gas pressure: 10 arb, capillary temperature: $350{ }^{\circ} \mathrm{C}$, heater temperature: $300{ }^{\circ} \mathrm{C}$, scan mode: full MS (Mass spectrometry) (resolution 70,000), and scan range: $\mathrm{m} / \mathrm{z}$ 100-1,500. The data collected by high-resolution liquid quality were collected by CD2.1 (Thermo Fisher), and then the database was retrieved and compared (mzCloud, mzVault, ChemSpider).

\section{Lysosome staining}

RAW64.7 cells and LPM $\left(100 \mu \mathrm{L} /\right.$ well, $\left.1.0 \times 10^{5} / \mathrm{mL}\right)$ were cultured in 96-well plates for $24 \mathrm{~h}$. Lysosome staining was detected with the lysosome staining kit (Abnova, KA4111, Taibei City, Taiwan, China). When cells were properly fused, the cells were transferred to the corresponding culture medium and $100 \mu \mathrm{L}$ of Lyso Green working solution $(20 \mu \mathrm{L}$ of $500 \times$ Lyso Green stock solution in $10 \mathrm{~mL}$ of live cell staining buffer) was added as described previously (8). The cells were incubated at $37^{\circ} \mathrm{C}$ in an atmosphere containing $5 \% \mathrm{CO}_{2}$ for $1 \mathrm{~h}$. Finally, the cells were visualized under a fluorescence microscope with a fluorescein isothiocyanate (FITC) filter set (excitation and emission at 490 and $525 \mathrm{~nm}$, respectively).

\section{Lysosome activity}

Lysosome activity was assayed as described previously (24). Briefly, RAW264.7 cells were solubilized in $25 \mu \mathrm{L}$ of $0.1 \%$ Triton X-100. Next, the lysates were incubated with $150 \mu \mathrm{L}$ of $10 \mathrm{mM}$ p-nitrophenyl phosphate (Sigma, USA) for $1 \mathrm{~h}$ at $37^{\circ} \mathrm{C}$. The reaction was stopped by adding $50 \mu \mathrm{L}$ of $0.2 \mathrm{M}$ borate buffer, and absorbance of the mixture at $405 \mathrm{~nm}$ was determined using a spectrophotometer. Relative lysosome activity (\%) was calculated as the ratio of the absorbance at $405 \mathrm{~nm}$ of TCXYT-treated cells to that of control cells multiplied by $100 \%$.

\section{Immunofluorescence}

Colonic tissue or treated cells were fixed with 4\% (w/v) paraformaldehyde (Sigma, USA), and blocked and incubated with an anti-CD11c antibody $(1: 100)$ overnight at $4{ }^{\circ} \mathrm{C}$. The cells were then washed in phosphate-buffered saline (PBS). After incubation with a secondary FITC-conjugated antibody and 4',6-diamidino-2-phenylindole (DAPI; Sigma, USA), the cells were rewashed in PBS, mounted in anti-fade reagent, and observed under an Olympus microscope, as described previously $(25,26)$.

\section{Statistical analysis}

Data were analyzed using IBM SPSS version 21.0 (IBM Corp., Armonk, NY, USA) and were presented as mean \pm standard error of mean. One-way analysis of variance or a general linear model with repeated measures was used to analyze the data of $\geq 3$ groups, and the least significant difference post-hoc test was used for multiple comparisons. Student's $t$-test was used to analyze differences between two groups. $\mathrm{P}<0.05$ indicated statistical significance.

TNBS-induced chronic colitis, the disease activity index (DAI), ELISA, histological analysis and Western blot are described elsewhere $(27,28)$.

The isolation of colonic LPM, cell culture, cell viability, and proliferation have been described elsewhere (29-31).

\section{Results}

\section{UPLC/UV chromatograms of the TCXYT extract}

The TCXYT extract was separated within $20 \mathrm{~min}$ on a C18 column $(150 \times 2.1 \mathrm{~mm} 1.8 \mu \mathrm{m})$ eluted with acetonitrile, methanol, and water containing $0.1 \%$ formic acid. The compounds were detected in the (-) ESI mode, and their MS/MS spectra were analyzed in an untargeted manner. Although TCXYT exhibited only a few major peaks in the HPLC/UV analysis, a number of minor compounds could be observed in the enlarged chromatogram (Figure 1). A total of 174 compounds were identified from TCXYT (Table S2). The chemical analysis of TCXYT extract served as the quality control for the reproducibility of the animal experiment.

\section{TCXYT significantly ameliorates inflammation and colonic mucosal injury}

After the colitis model was established, TCXYT administration was initiated at day 3 for 7 consecutive days (Figure $2 A$ ). Compared with the control group, colonic mucosal injury was aggravated in the model group, as evidenced by increased DAI, IL- $1 \beta$, and TNF- $\alpha$ serum levels, and decreased IL-4 and IL-10 levels (Figure 2B,C,D,E,F,G). Treatment with TCXYT for 7 days significantly ameliorated injury to the colonic mucosa (Figure 2G). TCXYT was also found to significantly decrease 


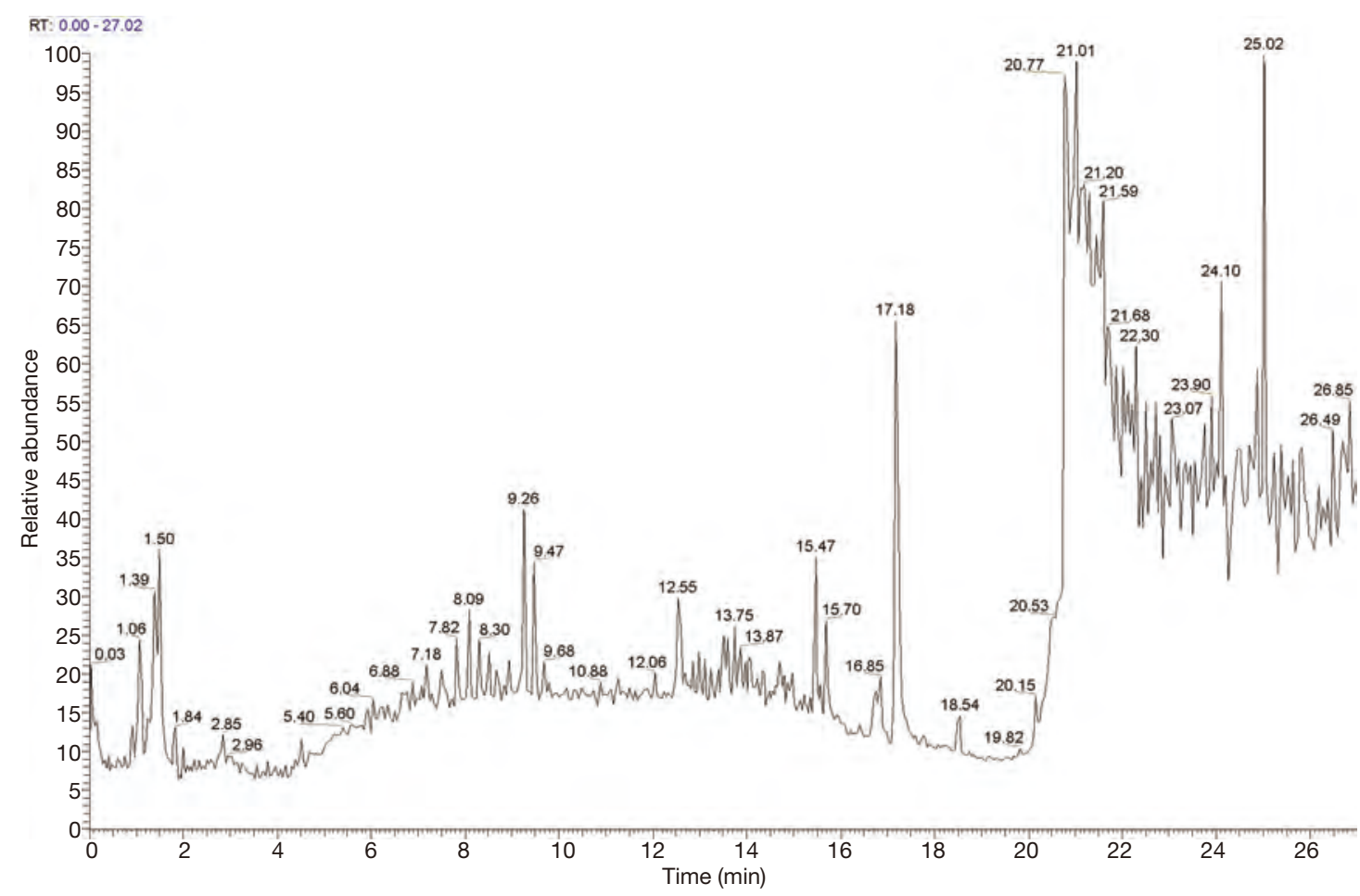

Figure 1 Ultra-high-performance liquid chromatography/UV of Tiaochang Xiaoyan tablet (TCXYT) extract (270 nm).

the DAI and the level of serum pro-inflammatory cytokines in a dose-dependent manner (Figure 2B,C,D), and increase the level of anti-inflammatory cytokines (Figure $2 E, F$ ).

\section{TCXYT reduces the infiltration of $C D 11 c^{+}$macrophages in colonic mucosa}

Compared with the control group, the degree of CD11 $\mathrm{c}^{+}$ macrophage infiltration in the model group was more severe (Figure $3 A$ ), and the number of CD11 $\mathrm{c}^{+}$cells in the lamina propria was greater (Figure 3B). Compared with the model group, the infiltration degree and number of CD11 $\mathrm{c}^{+}$ macrophages in the high-, middle-, and low-dose TCXYT groups and the HS group decreased. The TCXYT group was dose dependent, and there was no significant difference between the middle-dose group and the high-dose group (Figure $3 A, B$ ). In short, TCXYT can reduce the infiltration of $\mathrm{CD} 11 \mathrm{c}^{+}$macrophages and the number of CD11 $\mathrm{c}^{+}$cells in the lamina propria in rats with chronic colitis.

\section{TCXYT promoted the activation of lysosome in LPM}

The number and activity of lysosomes in LPM in the experimental colitis groups decreased (Figure $4 A, B, C$ ). After
7 days of treatment with TCXYT, the number and activity of lysosomes in LPM significantly increased compared with those in the model group (Figure 4A,B,C). In addition, TCXYT regulated the lysosomal activity in LPM in a dose-dependent manner (Figure $4 C$ ). The expression of LC3B in LPM significantly decreased in the colitis groups compared with the control group (Figure $4 D$ ). However, after 7 days of treatment with TCXYT, the expression of LC3B in LPM significantly increased compared with the model group. These effects were more significant in the medium- and high-dose groups than in the low-dose group (Figure 4A,B,C,D).

\section{TCXYT increases the viability, but not the proliferation, of RAW264.7 cells}

The viability of RAW264.7 cells was reduced, and their proliferation was reduced significantly, by LPS+IFN- $\gamma$ (Figure $5 A, B$ ). However, the viability of RAW264.7 cells increased significantly with TCXYT in a dose-dependent manner (Figure 5C), and their proliferation was unaffected by treatment with TCXYT for 24 and $48 \mathrm{~h}$ (Figure $5 D$ ). TCXYT also significantly increased the viability of RAW264.7 cells stimulated with LPS $(10 \mu \mathrm{g} / \mathrm{mL})$ plus 
A

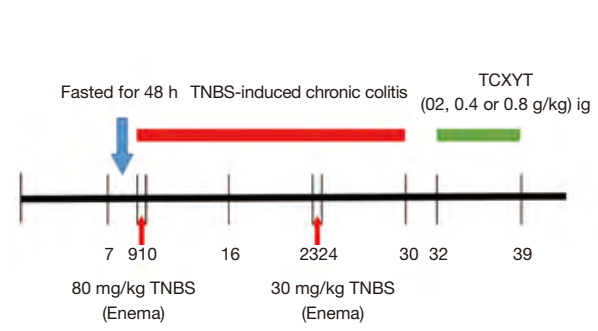

D

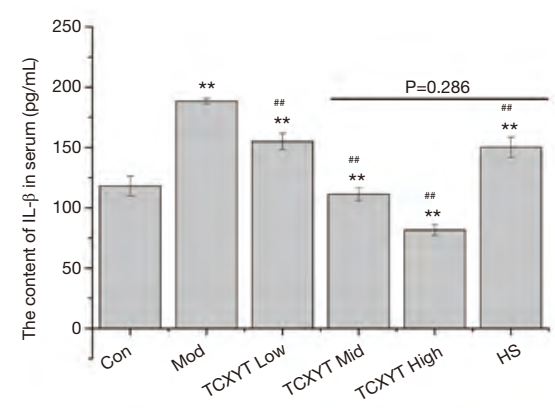

G

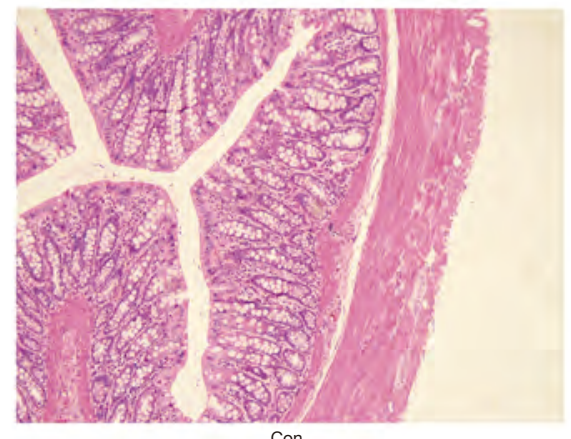

Con

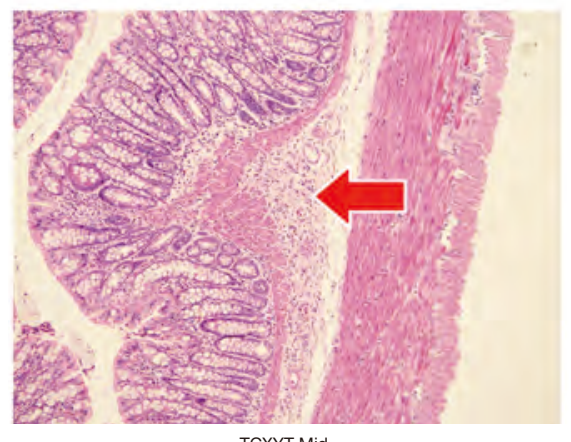

B

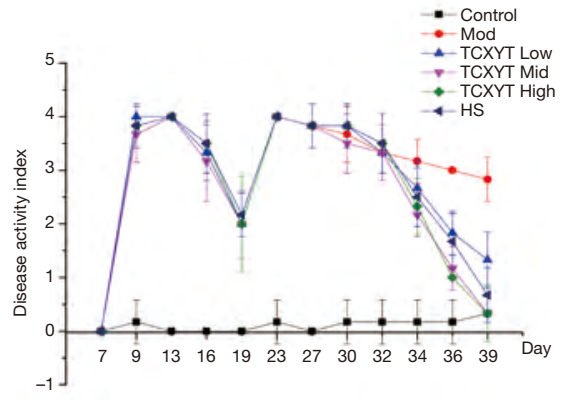

E
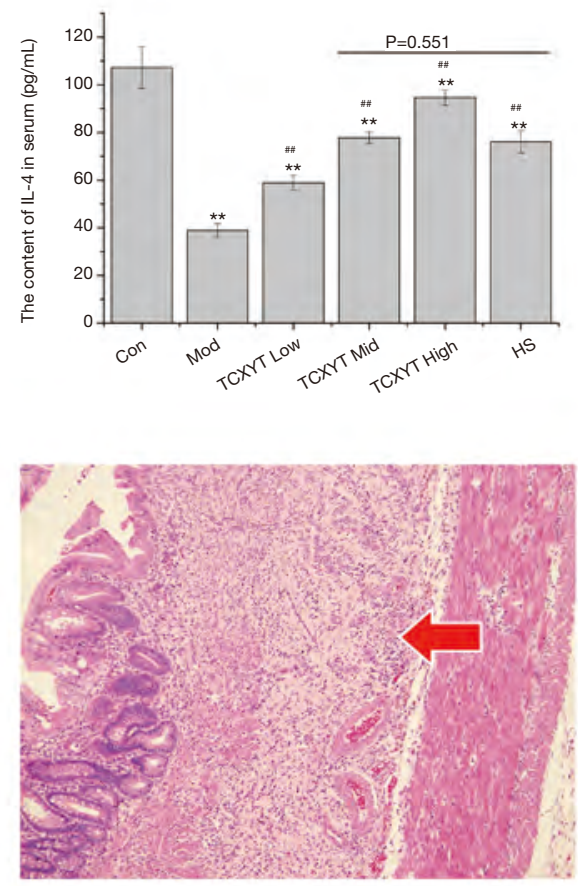

Mod

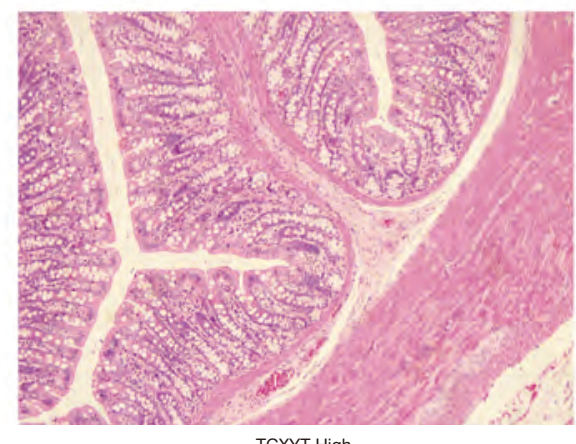

C

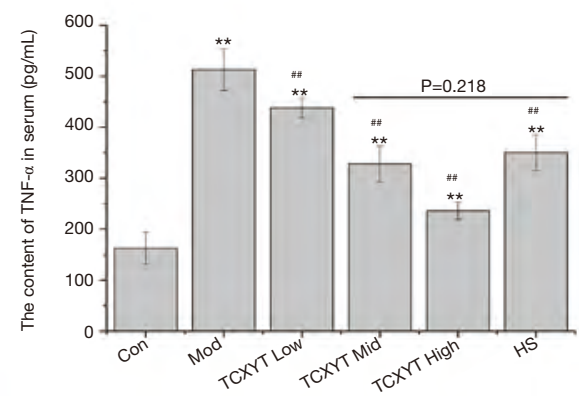

$\mathrm{F}$
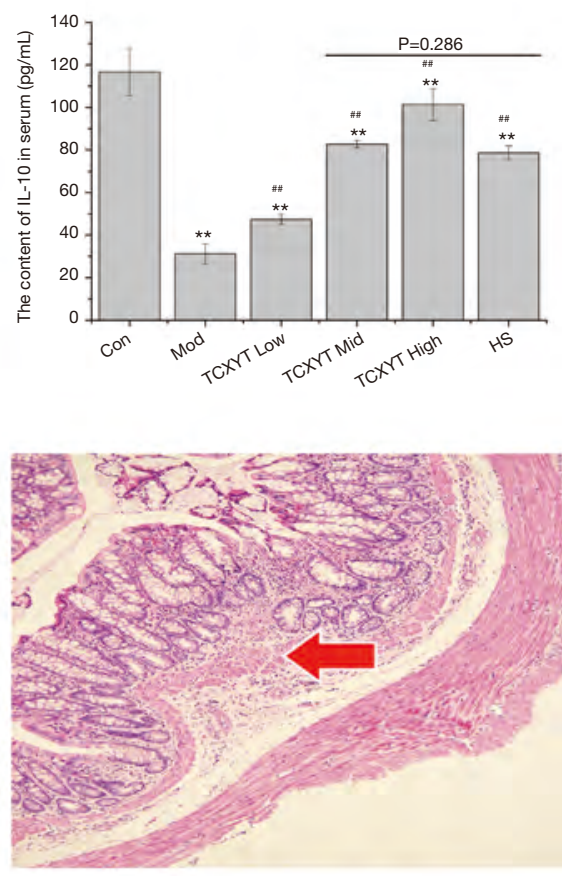

TCXYT Low

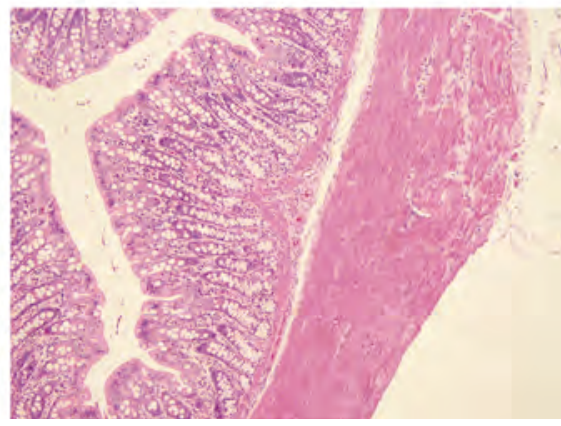

HS

Figure 2 Tiaochang Xiaoyan tablet (TCXYT) ameliorates inflammation and colonic mucosal injury. (A) TCXYT administration was initiated at day 3 for 7 consecutive days. (B,C,D,E,F) Colonic mucosal injury; daily activity index (DAI) scores; and interleukin (IL)-1 $\beta$, tumor necrosis factor- $\alpha$, IL-4, and IL-10 levels. (G) Histopathological analysis of colon tissue (magnification $\times 400$ ). The red arrows indicate colonic mucosal inflammatory injuries. Data are presented as mean \pm standard error of mean of 3 independent experiments performed in triplicate. **, $\mathrm{P}<0.01$ vs. control; ;\#, $\mathrm{P}<0.01$ vs. model. Con: Control group; Mod: Model group; TCXYT Low, Mid and High: low-, middle-, and highdose TCXYT; HS: HS group (treated with HS). 


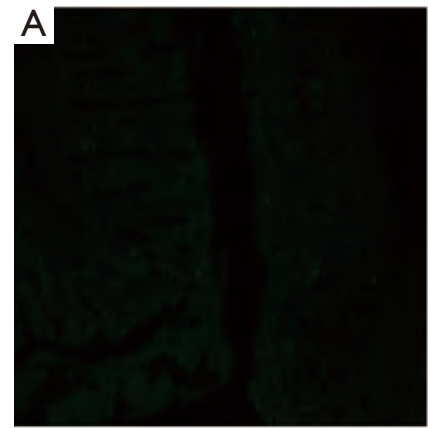

Con

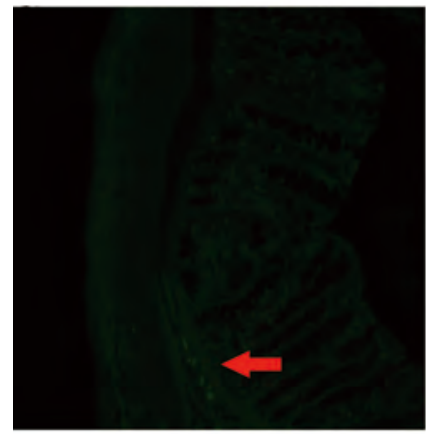

TCXYT Mid

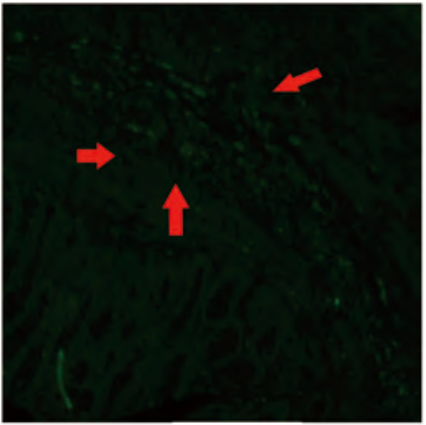

Mod

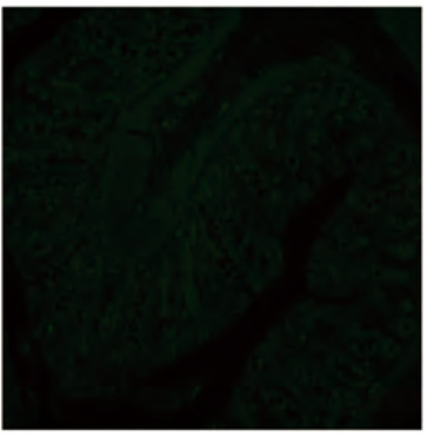

TCXYT High

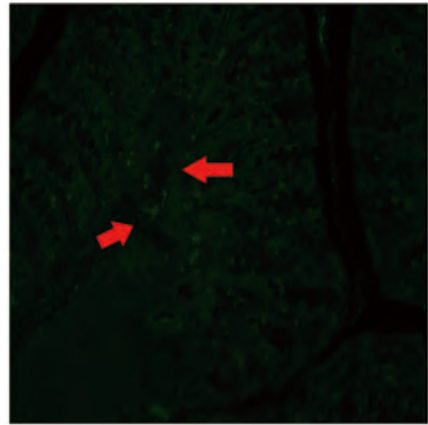

TCXYT Low

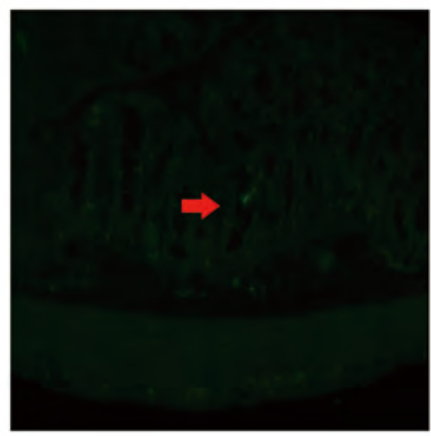

HS

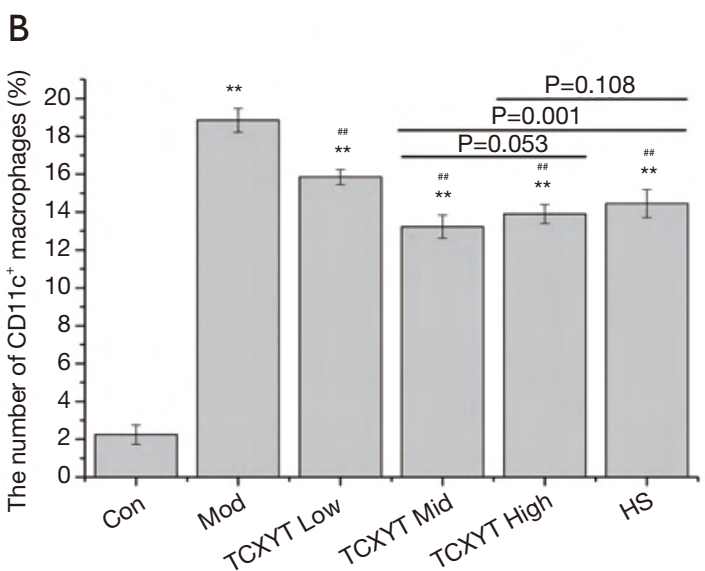

Figure 3 Tiaochang Xiaoyan tablet (TCXYT) reduces CD11 $\mathrm{c}^{+}$macrophage infiltration in the colonic mucosa. (A) Infiltration of CD11 $\mathrm{c}^{+}$ macrophages (CD11 ${ }^{+}$macrophages was showed by the arrows, magnification $\left.\times 400\right)$; (B) number of CD11 $\mathrm{c}^{+}$lamina propria macrophages in colon tissue. Data are presented as mean \pm standard error of mean of 3 independent experiments performed in triplicate. One-way analysis of variance and Student's $t$-test. ${ }^{* *}, \mathrm{P}<0.01$ vs. control; ${ }^{\# \#}, \mathrm{P}<0.01$ vs. model. The red arrows indicate CD11 $c^{+}$lamina propria macrophages. Con: Control group; Mod: Model group; TCXYT Low, Mid and High: low-, middle-, and high-dose TCXYT; HS: HS group (treated with HS).

IFN- $\gamma(10 \mathrm{ng} / \mathrm{mL})$ in a dose-dependent manner, but did not influence their proliferation. On the basis of this, we used TCXYT at $0.12,0.06$, and $0.03 \mathrm{~g} / \mathrm{mL}$ in subsequent experiments.

\section{TCXYT activates lysosomes in $R A W 264.7$ cells}

The number and activity of lysosomes significantly increased with TCXYT compared with the control group in a dose-dependent manner (Figure $6 A, B, C$ ). 


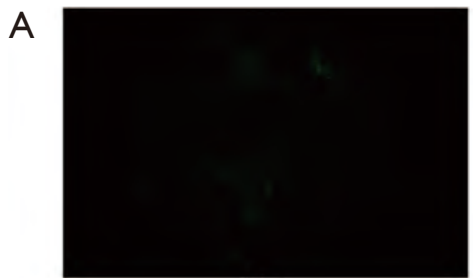

Con

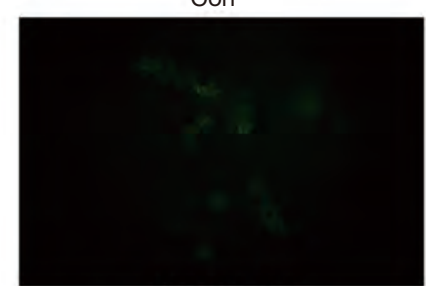

TCXYT Mid

B

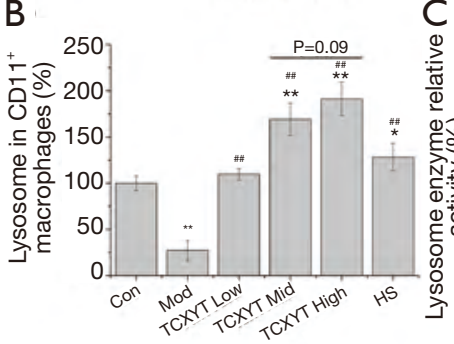

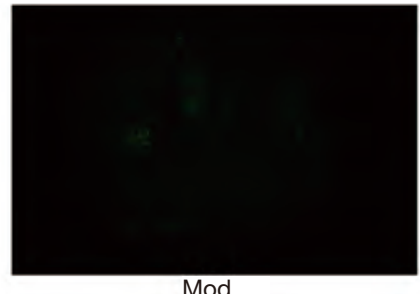

Mod

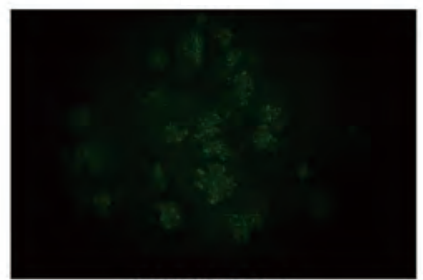

TCXYT High

C

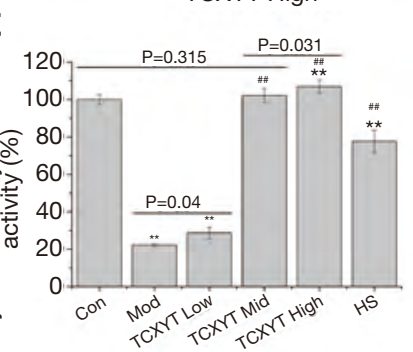

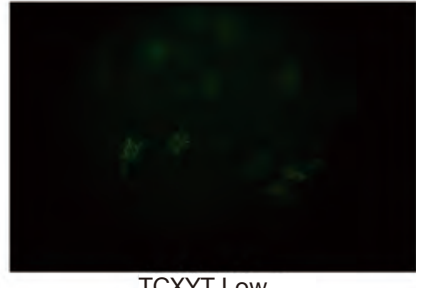

TCXYT Low

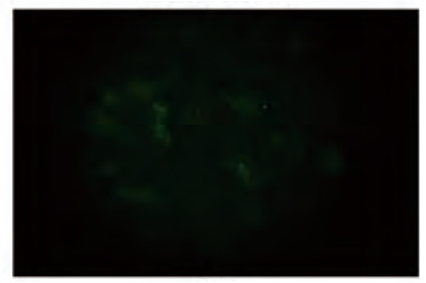

D
HS

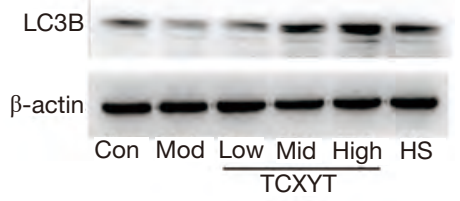

Figure 4 Tiachang Xiaoyan tablet (TCXYT) promotes the activation of lysosomes in (in vitro). (A) Fluorescence images of lysosomes in lamina propria macrophages (LPM) (magnification ×200); (B) ratio of the number of lysosome in LPM; (C) lysosomal activity in LPM; (D) expression of LC3B in LPs. Data are presented as mean \pm standard error of mean of 3 independent experiments performed in triplicate. **, $\mathrm{P}<0.01$ vs. control; ${ }^{\#}, \mathrm{P}<0.01$ vs. model. Con: Control group; Mod: Model group; Low, Mid and High: low-, middle-, and high-dose TCXYT; HS: HS group (treated with HS).

The effects in the middle- and high-dose groups were similar and were superior to those of the low-dose group (Figure $6 A, B)$. However, the expression of LC3B in RAW264.7 cells did not differ markedly among the treatment groups (Figure 6D).

TCXYT also significantly increased the number and activity of lysosomes in RAW264.7 cells stimulated with LPS+IFN- $\gamma$ in a dose-dependent manner compared with cells treated with only LPS+IFN- $\gamma$ (Figure 6C,E,F). The expression of LC3B in RAW264.7 cells treated with LPS+IFN- $\gamma$ was lower than that in the control group, but increased with TCXYT treatment (Figure 6G). However, there was no significant difference between the mediumand high-dose groups.

Therefore, TCXYT ameliorated inflammation in rats with chronic colitis, possibly by increasing the number and activity of lysosomes in macrophages.

\section{TCXYT regulates the TLR9/MyD88/IRAK signaling patbway}

The TLR9 signaling pathway plays a central role in the regulation of mucosal innate immunity, particularly of macrophage autophagy, which is implicated in the pathogenesis of UC (13). In the present study, the TLR9, MyD88, IRAK1, and IRAK4 protein levels in LPM were significantly increased in the groups with colitis compared with the control group. TCXYT significantly decreased the TLR9, MyD88, IRAK1, and IRAK4 protein levels in LPM in rats with colitis in a dose-dependent manner. In addition, the effects in the medium- and high-dose groups were superior to those in the low-dose group (Figure 7A).

The TLR9, MyD88, IRAK1, and IRAK4 protein levels in RAW264.7 cells treated with LPS+IFN- $\gamma$ were significantly increased compared with those in the control group. TCXYT significantly decreased the TLR9, MyD88, 
A

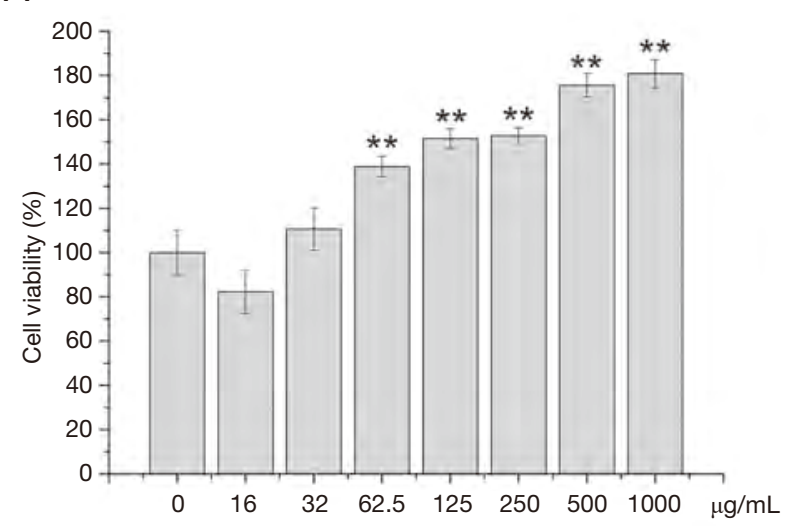

C

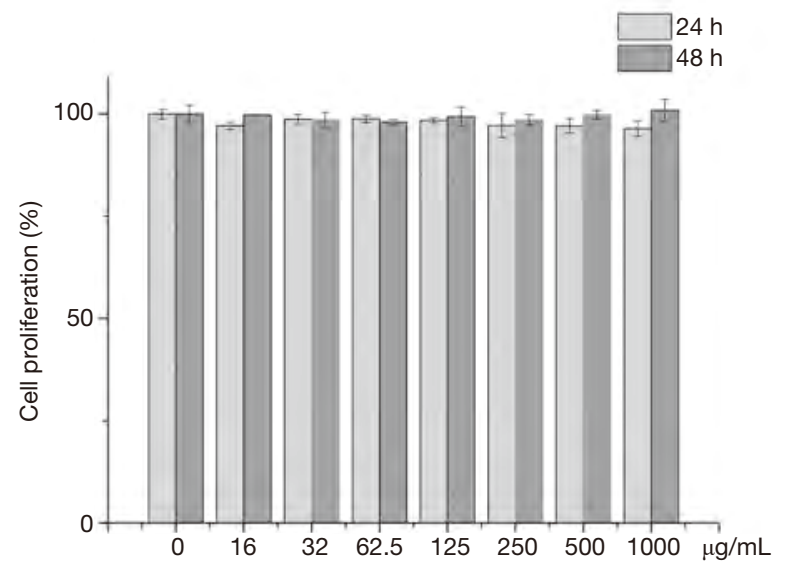

B
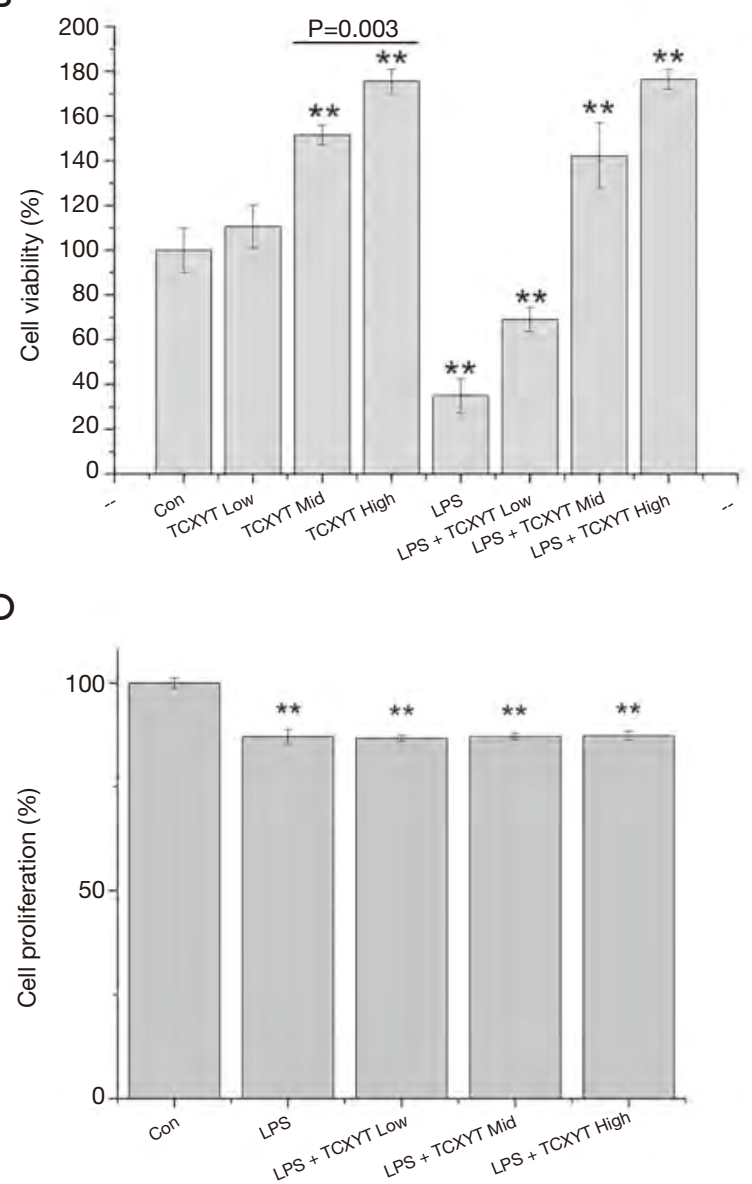

Figure 5 Tiaochang Xiaoyan tablet (TCXYT) reduces the viability, but not the proliferation, of RAW264.7 cells. (A) Viability of RAW264.7 cells at different concentrations; (B) RAW264.7 cell relative viability in different stimulation groups; (C) proliferation of RAW264.7 cells at different concentrations; (D) RAW264.7 cell relative proliferation in different stimulation groups. Data are presented as mean \pm standard error of mean of 3 independent experiments performed in triplicate. ${ }^{* *}, \mathrm{P}<0.01$ ss. control. Con: Control group; LPS: LPS group (stimulated with LPS); TCXYT Low, Mid and High: low-, middle-, and high-dose TCXYT.

IRAK1, and IRAK4 protein levels in a dose-dependent manner (Figure $7 B$ ).

\section{Inbibition of the TLR9 signaling patbway ameliorates inflammation and activates lysosomes}

HS, an inhibitor of autophagy and TLR7/9, is an antimalarial agent that is also used to treat inflammatory conditions (32). When RAW264.7 cells stimulated with LPS+IFN- $\gamma$ were treated with HS for $24 \mathrm{~h}$, the TLR9, MyD88, IRAK1, and IRAK4 protein levels significantly decreased, and the LC3B protein level increased, compared with cells stimulated with LPS+IFN- $\gamma$ (Figure 8A). Similarly, after treatment with HS for 7 days, the TLR9, MyD88, IRAK1, and IRAK4 protein levels in LPM significantly reduced compared with those in the model group (Figure 8B). HS treatment also increased the expression of LC3B and the lysosomal number and activity in LPM (Figure $4 A, B, C, D$ ). Inflammation and colonic mucosal injury were also significantly ameliorated compared with the model group (Figure 2C,E,F,G).

\section{Discussion}

TCXYT is a herbal preparation that suppresses inflammation in the colonic mucosa of patients with UC (19). The 
A

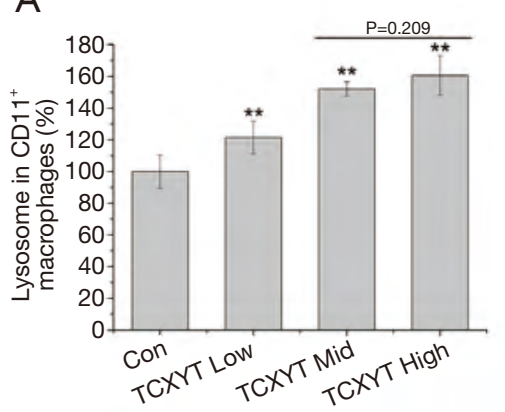

D

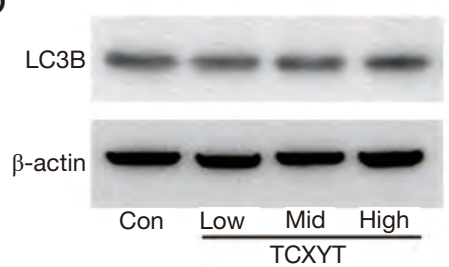

$\mathrm{F}$

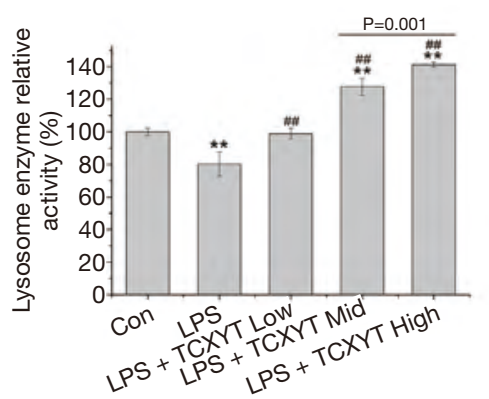

B

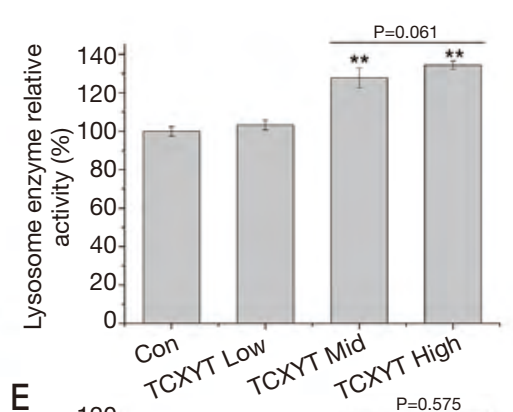

$\mathrm{E}$
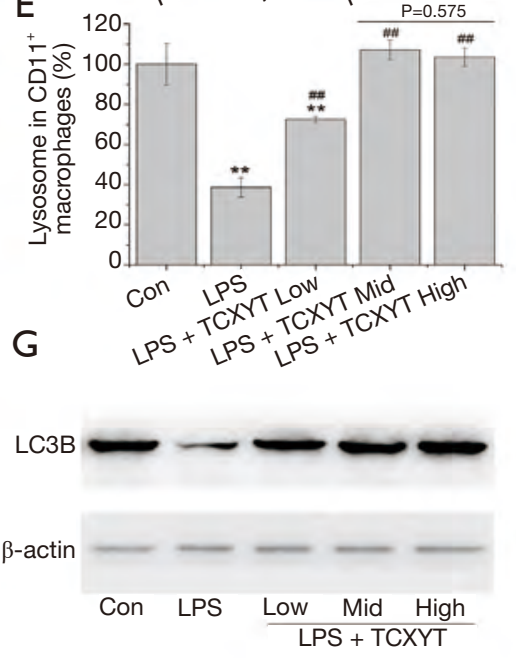

C

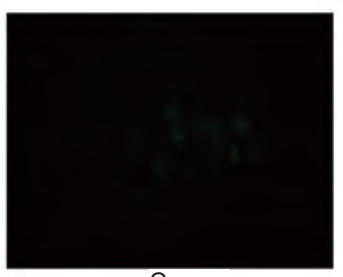

Con
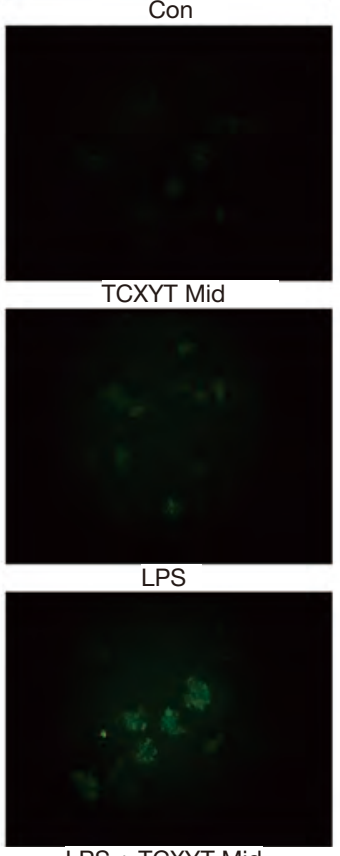

LPS + TCXYT Mid

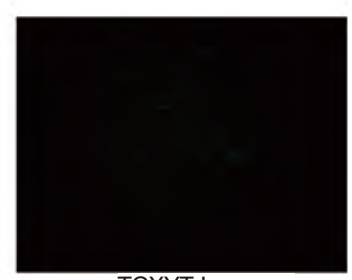

TCXYT Low

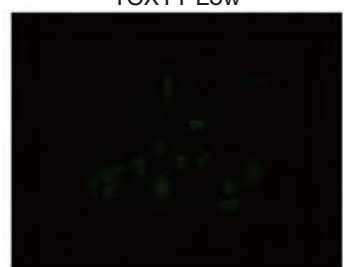

TCXYT High

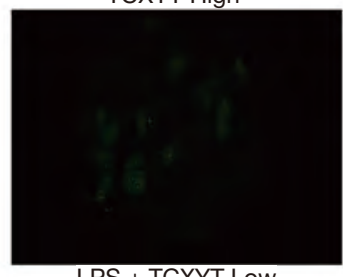

LPS + TCXYT Low

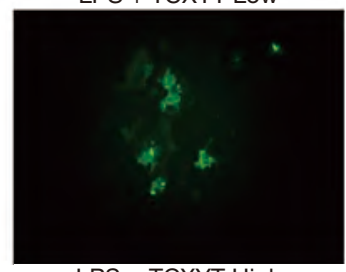

LPS + TCXYT High

Figure 6 Effects of Tiaochang Xiaoyan tablet (TCXYT) on lysosomes in RAW264.7 cells. (A) Effects of low-, middle-, and high-dose TCXYT on the number of lysosomes in RAW264.7 cells; (B) effects of low-, middle-, and high-dose TCXYT on the lysosomal activity in RAW264.7 cells; (C) effects of low-, middle-, and high-dose TCXYT on the number of lysosomes in RAW264.7 cells stimulated by lipopolysaccharide (LPS)+interferon- $\gamma($ IFN- $\gamma$ ) (magnification $\times 200)$; (D) fluorescence micrographs of lysosome activity in RAW264.7 cells stimulated by LPS+INF- $\gamma$; (E) effects of low-, middle-, and high-dose TCXYT on the expression of LC3B in RAW264.7 cells; (F) effects of low-, middle-, and high-dose TCXYT on the lysosomal activity in RAW264.7 cells stimulated by LPS+INF- $\gamma$; (G) effects of low-, middle-, and high-dose TCXYT on the expression of LC3B in RAW264.7 cells stimulated by LPS+INF- $\gamma$ Data are presented as mean \pm standard error of mean of 3 independent experiments performed in triplicate. ${ }^{* *}, \mathrm{P}<0.01$ vs. control; ${ }^{\# \#}, \mathrm{P}<0.01$ vs. model. Con: Control group; LPS: LPS group (stimulated with LPS); TCXYT Low, Mid and High: low-, middle-, and high-dose TCXYT.

bioactive components of TCXYT and their contents vary in composition and extraction methods. To clarify the chemical composition and to evaluate the quality of TCXYT, we used HPLC/UV to analyze the composition of TCXYT used in the present study. The components of $T C X Y T$ were detected, indicating that $T C X Y T$ is a mixture of compounds. The components and their contents are in line with the provisions of Pharmacopoeia of the People's Republic of China and meet the requirements of good clinical practice in China.
Macrophages, a type of myeloid cell, play a pivotal role in the innate immune response against pathogens. In patients with $\mathrm{UC}$, a heterogeneous population of inflammatory cells is present in colon tissues, particularly CD $11 \mathrm{c}^{+}$macrophages $(33,34)$. Although the role of macrophages in the development of UC has been explored, the effect and its mechanism are unclear. However, it is well known that increased macrophage infiltration in colon tissue and abnormal polarization are related to the development of inflammation in UC (33). In the present 
A

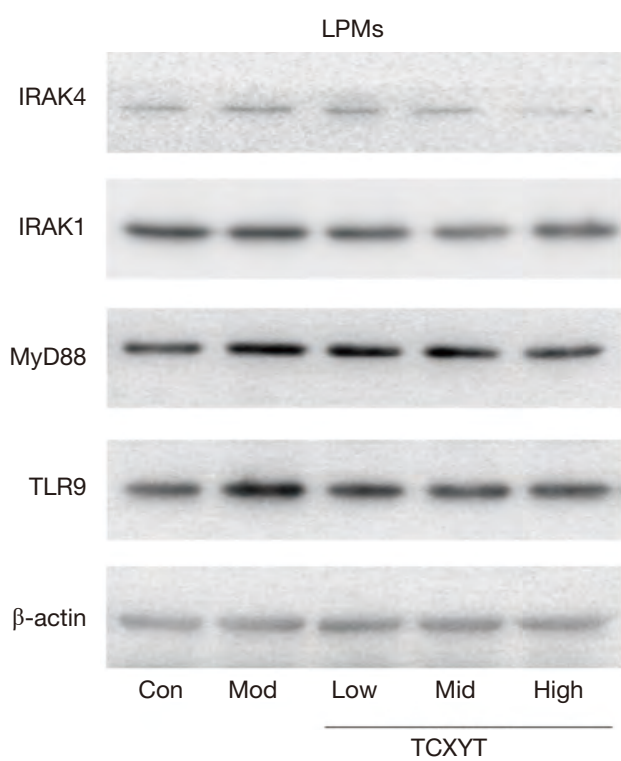

B
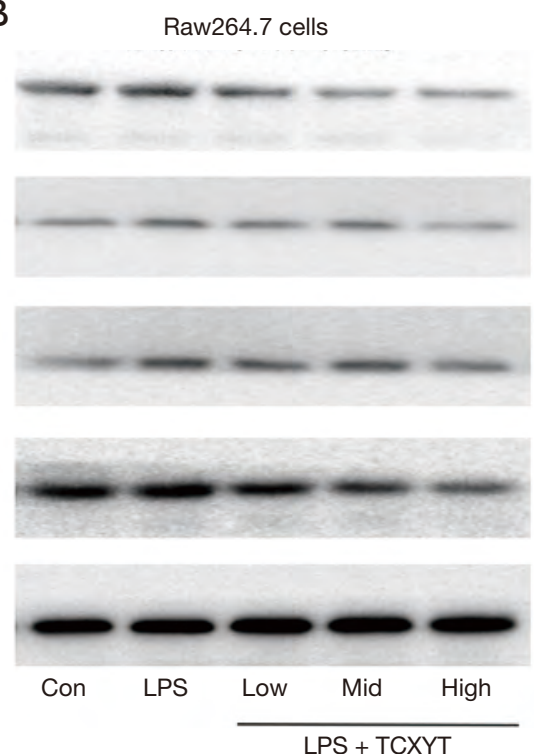

Figure 7 Tiaochang Xiaoyan tablet (TCXYT) regulates the Toll-like receptor 9 (TLR9)/myeloid differentiation primary response 88 (MyD88)/ interleukin receptor-associated kinase (IRAK) signaling pathway. (A) TLR9, MyD88, IRAK1, and IRAK4 protein levels in lamina propria macrophages were significantly upregulated in the experimental colitis groups and significantly decreased by $T C X Y T$ in a dose-dependent manner. (B) TLR9, MyD88, IRAK1, and IRAK4 protein levels in RAW264.7 cells treated with lipopolysaccharide + interferon- $\gamma$ were significantly increased compared with the control group, and were significantly decreased with TCXYT in a dose-dependent manner. Con: Control group; Mod: Model group; LPS: LPS group (stimulated with LPS); TCXYT Low, Mid and High: low-, middle-, and high-dose TCXYT.

study, compared with the control group, the infiltration of $\mathrm{CD} 11 \mathrm{c}^{+}$macrophages in the colon of rats with chronic colitis was significantly increased, and the mucosal damage was aggravated. After 7 days of TCXYT treatment, the degree of $\mathrm{CD}_{1} 1^{+}$macrophage infiltration and tissue injury in the colon tissue of the high-, middle-, and low-dose TCXYT groups improved at different degrees. Therefore, $T C X Y T$ alleviates inflammation and promotes the repair of colon mucosal injury by reducing the infiltration of CD11c ${ }^{+}$ macrophages in the colon.

Lysosomes in macrophages are not only degradative organelles but also play a central role in nutrient sensing, metabolism, and cell-growth regulation (35). Lysosomal number and activity, two important elements of lysosome function, are related to the activity and polarization of macrophages $(36,37)$. The lysosomal activity of CD11 $\mathrm{c}^{+}$ macrophages in the colon of patients with $\mathrm{UC}$ was significantly inhibited (38), but the mechanism is unknown. The number and activity of lysosomes in LPM isolated from the colon tissue of rats with chronic colitis significantly decreased, the level of pro-inflammatory cytokines increased, and anti-inflammatory cytokines decreased. In addition, TCXYT significantly increased the number and activity of lysosomes in CD11 $\mathrm{c}^{+}$macrophages. However, $T C X Y T$ increased the viability, but not the proliferation, of $\mathrm{CD} 11 \mathrm{c}^{+}$macrophages in vitro, suggesting that its antiinflammatory activity was mediated by effects on lysosomes in macrophages, rather than on macrophage proliferation directly; however, the mechanism is unclear.

TLRs play central roles in the regulation of macrophagemediated mucosal innate immunity and in the pathogenesis of UC (31). TLR9 is a conserved transmembrane receptor that recognizes pathogen-associated molecular patterns, and initiates an immune response by modulating lysosomal activity in macrophages (39). TLR9 is an important component of the TLR9/MyD88/IRAK signaling pathway, and its activation triggers the production of cytokines and chemokines, which are important in the development of inflammation in UC $(31,40)$. In addition, the lysosomal activity in macrophages is regulated by the TLR9/ MyD88 signaling pathway in macrophages (41). IRAK1 and IRAK4 are important factors in the TLR9/MyD88 signaling pathway and play a key role in the activation of macrophages $(42,43)$. However, the role of IRAKs (i.e., 


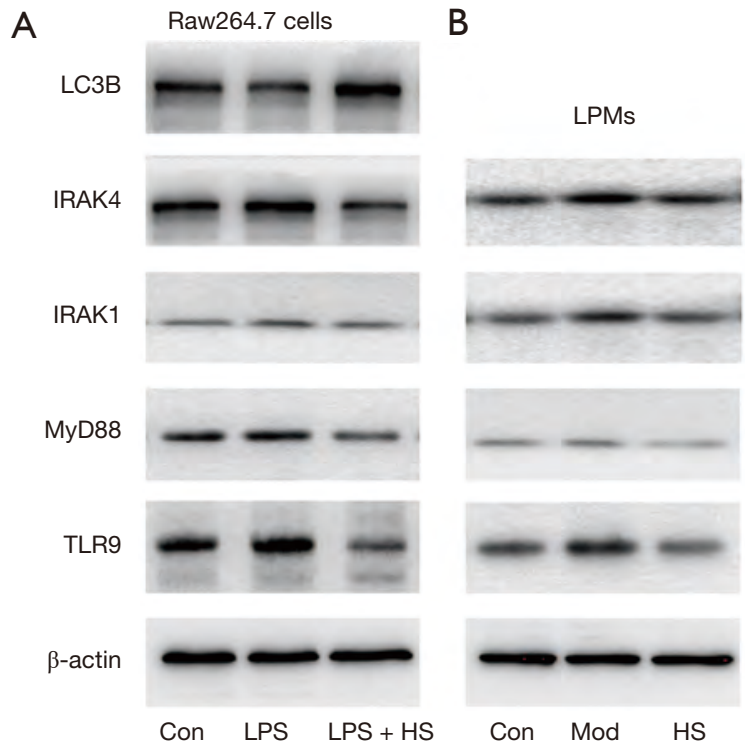

Figure 8 Inhibition of the Toll-like receptor 9 (TLR9) signaling pathway ameliorates inflammation and activates lysosomes in macrophages. (A) In RAW264.7 cells stimulated with lipopolysaccharide (LPS) + interferon- $\gamma($ IFN- $\gamma$ ) and treated with hydroxychloroquine sulfate (HS) for $24 \mathrm{~h}$, the TLR9, myeloid differentiation primary response 88 (MyD88), interleukin receptorassociated kinase (IRAK) 1, and IRAK4 protein levels significantly decreased compared with those in cells only stimulated with LPS+IFN- $\gamma$. (B) TLR9, MyD88, IRAK1, and IRAK4 protein levels in lamina propria macrophages treated with HS significantly decreased compared with those in the model group. Con: Control group; Mod: Model group; LPS: LPS group (stimulated with LPS).

IRAK1 and IRAK4) in macrophages in the development of UC is unknown. We found that lysosomal activity in macrophages was inhibited in RAW264.7 cells stimulated with LPS+IFN- $\gamma$, and in LPM from the colons of rats with chronic colitis, the IRAK1 and IRAK4 protein levels increased significantly and the TLR9/MyD88 signaling pathway was activated. However, the IRAK1 and IRAK4 protein levels significantly decreased and lysosomal activity in macrophages significantly increased with HS-mediated suppression of the TLR9/MyD88 signaling pathway. Therefore, the lysosomal activity in macrophages may be inhibited by activating the TLR9/MyD88/IRAK signaling pathway. In addition, TCXYT reduced the infiltration of CD $11 \mathrm{c}^{+}$macrophages in colon tissue and increased lysosomal activity in macrophages by inhibiting the TLR9/ MyD88/IRAK signaling pathway.

\section{Conclusions}

The composition and stability of TCXYT are elevated by chromatography. In the study, the results showed that $T C X Y T$ can be used as a qualified clinical drug which had stable drug composition and physical-chemical properties. In addition, TCXYT is promising for the treatment of $\mathrm{UC}$, as it ameliorates inflammation and $\mathrm{CD} 11 \mathrm{c}^{+}$macrophage infiltration in the colon of rats with chronic colitis. TCXYT may promote the activation of lysosomes in macrophages by inhibiting the TLR9/MyD88/IRAK signaling pathway. However, several issues warrant further study, including how lysosomes regulate the differentiation of macrophages and determination of the exogenous regulator of macrophages in the development of UC. These issues must be resolved if TCXYT is to be used to ameliorate inflammation in the colon mucosa of patients with UC.

\section{Acknowledgments}

The authors thank the technical staff for their assistance with the study and Dr. Zhaoyu Lu who reviewed the article. Funding: This work was supported by the National Natural Science Foundation of China (Nos. 81573786 and 81904106) and Young Creative Talents Project of Guangdong Province Universities and Colleges (No. 2018KQNCX044).

\section{Footnote}

Reporting Checklist: The authors have completed the ARRIVE reporting checklist. Available at http://dx.doi. org/10.21037/apm-21-250

Data Sharing Statement: Available at http://dx.doi. org/10.21037/apm-21-250

Conflicts of Interest: All authors have completed the ICMJE uniform disclosure form (available at http://dx.doi. org/10.21037/apm-21-250). The authors have no conflicts of interest to declare.

Ethical Statement: The authors are accountable for all aspects of the work in ensuring that questions related to the accuracy or integrity of any part of the work are appropriately investigated and resolved. The Institutional Animal Care and Use Committee of The Second Affiliated Hospital of Guangzhou University of Chinese Medicine 
approved all of the procedures involving the rats (animal ethics approval No. 2016021-2). Experiments were performed in compliance with the Institutional Animal Care and Use Committee of Guangzhou University of Chinese Medicine's guidelines for the care and use of animals.

Open Access Statement: This is an Open Access article distributed in accordance with the Creative Commons Attribution-NonCommercial-NoDerivs 4.0 International License (CC BY-NC-ND 4.0), which permits the noncommercial replication and distribution of the article with the strict proviso that no changes or edits are made and the original work is properly cited (including links to both the formal publication through the relevant DOI and the license). See: https://creativecommons.org/licenses/by-nc-nd/4.0/.

\section{References}

1. Ungaro R, Mehandru S, Allen PB, et al. Ulcerative colitis. Lancet 2017;389:1756-70.

2. Boal Carvalho P, Cotter J. Mucosal Healing in Ulcerative Colitis: A Comprehensive Review. Drugs 2017;77:159-73.

3. Olén O, Erichsen R, Sachs MC, et al. Colorectal cancer in ulcerative colitis: a Scandinavian population-based cohort study. Lancet 2020;395:123-31.

4. Cosin-Roger J, Simmen S, Melhem H, et al. Hypoxia ameliorates intestinal inflammation through NLRP3/ mTOR downregulation and autophagy activation. Nat Commun 2017;8:98.

5. Shah YM. The role of hypoxia in intestinal inflammation. Mol Cell Pediatr 2016;3:1.

6. Weigert A, Mora J, Sekar D, et al. Killing Is Not Enough: How Apoptosis Hijacks Tumor-Associated Macrophages to Promote Cancer Progression. Adv Exp Med Biol 2016;930:205-39.

7. Freire MO, Van Dyke TE. Natural resolution of inflammation. Periodontol 2000 2013;63:149-64.

8. Li J, Cui X, Ma X, et al. Recombinant Buckwheat Trypsin Inhibitor Improves the Protein and Mitochondria Homeostasis in Caenorhabditis elegans Model of Aging and Age-Related Disease. Gerontology 2019;65:513-23.

9. Jain N, Moeller J, Vogel V. Mechanobiology of Macrophages: How Physical Factors Coregulate Macrophage Plasticity and Phagocytosis. Annu Rev Biomed Eng 2019;21:267-97.

10. Fogarty CE, Bergmann A. The Sound of Silence: Signaling by Apoptotic Cells. Curr Top Dev Biol 2015;114:241-65.

11. Shi B, Huang QQ, Birkett R, et al. SNAPIN is critical for lysosomal acidification and autophagosome maturation in macrophages. Autophagy 2017;13:285-301.

12. Aflaki E, Moaven N, Borger DK, et al. Lysosomal storage and impaired autophagy lead to inflammasome activation in Gaucher macrophages. Aging Cell 2016;15:77-88.

13. McAlpine W, Sun L, Wang KW, et al. Excessive endosomal TLR signaling causes inflammatory disease in mice with defective SMCR8-WDR41-C9ORF72 complex function. Proc Natl Acad Sci U S A 2018;115:E11523-E11531.

14. Xia Y, Liu N, Xie X, et al. The macrophage-specific V-ATPase subunit ATP6V0D2 restricts inflammasome activation and bacterial infection by facilitating autophagosome-lysosome fusion. Autophagy 2019;15:960-75.

15. Figliuolo da Paz V, Jamwal DR, Gurney M, et al. Rapid Downregulation of DAB2 by Toll-Like Receptor Activation Contributes to a Pro-Inflammatory Switch in Activated Dendritic Cells. Front Immunol 2019;10:304.

16. Matharu KS, Mizoguchi E, Cotoner CA, et al. Tolllike receptor 4-mediated regulation of spontaneous Helicobacter-dependent colitis in IL-10-deficient mice. Gastroenterology 2009;137:1380-90 e1-3.

17. Yamamoto-Furusho JK, Gutierrez-Grobe Y, LopezGomez JG, et al. The Mexican consensus on the diagnosis and treatment of ulcerative colitis. Rev Gastroenterol Mex 2018;83:144-67.

18. Zhang Z, Cao H, Shen $\mathrm{P}$, et al. Ping weisan alleviates chronic colitis in mice by regulating intestinal microbiota composition. J Ethnopharmacol 2020;255:112715.

19. Beiping Z, Feng l, Suiping H, et al. Clinical study on treatment of Ulcerative colitis with Tiaochang Xiaoyan Tables combined with Changdiqing liquid enema. Pharmaceutical Industry Information 2005:61-2.

20. Cailing Z, Chun G, Shiying W, et al. Effect of Tiaochang Xiaoyan Tablets on Serum Cytokines in Patients with Mild-to-moderate Ulcerative Colitis. Journal of Guangzhou University of Traditional Chinese Medicine 2020;37:226-33.

21. Zhong CL, Guo C, Wang SY, et al. Effects of Tiao Chang Xiao Yan tables on serum cytokines and LC3B in rats with Ulcerative colitis. Acta Medica Mediterranea 2020;36:571-8.

22. Qiao X, Li R, Song W, et al. A targeted strategy to analyze untargeted mass spectral data: Rapid chemical profiling of Scutellaria baicalensis using ultra-high performance liquid chromatography coupled with hybrid quadrupole orbitrap mass spectrometry and key ion filtering. J Chromatogr A 2016;1441:83-95. 
23. Cao Y, Chen J, Wang Y, et al. HPLC/UV analysis of chlorfenapyr residues in cabbage and soil to study the dynamics of different formulations. Sci Total Environ 2005;350:38-46.

24. Chung CY, Yang WC, Liang CL, et al. Cytopiloyne, a polyacetylenic glucoside from Bidens pilosa, acts as a novel anticandidal agent via regulation of macrophages. J Ethnopharmacol 2016;184:72-80.

25. Yuan Z, Zhu X, Li Y, et al. Influence of iRoot SP and mineral trioxide aggregate on the activation and polarization of macrophages induced by lipopolysaccharide. BMC Oral Health 2018;18:56.

26. Uematsu S, Jang MH, Chevrier N, et al. Detection of pathogenic intestinal bacteria by Toll-like receptor 5 on intestinal CD11c+ lamina propria cells. Nat Immunol 2006;7:868-74.

27. Wang SY, Tao P, Hu HY, et al. Effects of initiating time and dosage of Panax notoginseng on mucosal microvascular injury in experimental colitis. World J Gastroenterol 2017;23:8308-20.

28. Wang YH, Ge B, Yang XL, et al. Proanthocyanidins from grape seeds modulates the nuclear factor-kappa B signal transduction pathways in rats with TNBS-induced recurrent ulcerative colitis. Int Immunopharmacol 2011;11:1620-7.

29. Shiying W, Boyun S, Jianye Y, et al. The Different Effects of VEGFA121 and VEGFA165 on Regulating Angiogenesis Depend on Phosphorylation Sites of VEGFR2. Inflamm Bowel Dis 2017;23:603-16.

30. Ichikawa N, Yamashita K, Funakoshi T, et al. Novel antiinflammatory agent 3-[(dodecylthiocarbonyl)-methyl]glutarimide ameliorates murine models of inflammatory bowel disease. Inflamm Res 2016;65:245-60.

31. Ungaro R, Fukata M, Hsu D, et al. A novel Toll-like receptor 4 antagonist antibody ameliorates inflammation but impairs mucosal healing in murine colitis. Am J Physiol Gastrointest Liver Physiol 2009;296:G1167-79.

32. Mortezagholi S, Babaloo Z, Rahimzadeh P, et al. Evaluation of TLR9 expression on PBMCs and CpG ODN-TLR9 ligation on IFN-alpha production in SLE patients. Immunopharmacol Immunotoxicol 2017;39:11-8.

33. Fuke N, Takagi T, Higashimura Y, et al. Lactobacillus brevis KB290 With Vitamin A Ameliorates Murine Intestinal Inflammation Associated With the Increase of CD11c+ Macrophage/CD103- Dendritic Cell Ratio. Inflamm Bowel Dis 2018;24:317-31.

34. Bernardo D, Marin AC, Fernandez-Tome S, et al. Human intestinal pro-inflammatory CD11c(high)CCR2(+)
CX3CR1(+) macrophages, but not their tolerogenic CD11c(-)CCR2(-)CX3CR1(-) counterparts, are expanded in inflammatory bowel disease. Mucosal Immunol 2018;11:1114-26.

35. Durso W, D'Autilia F, Amodeo R, et al. Probing labelinginduced lysosome alterations in living cells by imagingderived mean squared displacement analysis. Biochem Biophys Res Commun 2018;503:2704-9.

36. Robinet P, Ritchey B, Smith JD. Physiological difference in autophagic flux in macrophages from 2 mouse strains regulates cholesterol ester metabolism. Arterioscler Thromb Vasc Biol 2013;33:903-10.

37. Moheimani F, Kim CH, Rahmanto AS, et al. Inhibition of lysosomal function in macrophages incubated with elevated glucose concentrations: a potential contributory factor in diabetes-associated atherosclerosis. Atherosclerosis 2012;223:144-51.

38. Bauer C, Duewell P, Mayer C, et al. Colitis induced in mice with dextran sulfate sodium (DSS) is mediated by the NLRP3 inflammasome. Gut 2010;59:1192-9.

39. De Leo MG, Staiano L, Vicinanza M, et al. Autophagosome-lysosome fusion triggers a lysosomal response mediated by TLR 9 and controlled by OCRL. Nat Cell Biol 2016;18:839-50.

40. Uematsu S, Sato S, Yamamoto M, et al. Interleukin-1 receptor-associated kinase-1 plays an essential role for Toll-like receptor (TLR)7- and TLR9-mediated interferon-\{alpha\} induction. J Exp Med 2005;201:915-23.

41. Ewald SE, Lee BL, Lau L, et al. The ectodomain of Tolllike receptor 9 is cleaved to generate a functional receptor. Nature 2008;456:658-62.

42. Park SH, Baek SI, Yun J, et al. IRAK4 as a molecular target in the amelioration of innate immunity-related endotoxic shock and acute liver injury by chlorogenic acid. J Immunol 2015;194:1122-30.

43. Hirayama T, Tamaki Y, Takakubo Y, et al. Toll-like receptors and their adaptors are regulated in macrophages after phagocytosis of lipopolysaccharide-coated titanium particles. J Orthop Res 2011;29:984-92.

(English Language Editor: R. Scott)

Cite this article as: Wang S, Guo C, Zhang T, Zhong C, Zhao X, Su Y, Wei W, Zhang B. Tiaochang Xiaoyan extract tablets ameliorate chronic inflammation by activating macrophage lysosomes in chronic colitis rats. Ann Palliat Med 2021;10(2):2203-2216. doi: 10.21037/apm-21-250 
Supplementary

Table S1 Herbal composition of Tiaochang Xiaoyan tablet

\begin{tabular}{lllll}
\hline Latin name & English name & Chinese name & Dosage & Place of origin \\
\hline $\begin{array}{l}\text { Radix Astragali seu } \\
\text { Hedysari. }\end{array}$ & Milkvetch root & Huang-Qi & $15 \mathrm{~g}$ & Nei Mongol, China \\
$\begin{array}{l}\text { Radix Linderae } \\
\text { Combined spicebush } \\
\text { root }\end{array}$ & Wu-Yao & $15 \mathrm{~g}$ & Hunan, China \\
$\begin{array}{l}\text { Rhizoma Coptidis } \\
\text { Coptis Root }\end{array}$ & Huang-Lian & $10 \mathrm{~g}$ & Sichuan, China \\
Semen Hedyotis & Hedyotis & Bai hua she she cao & $20 \mathrm{~g}$ & Hunan, China \\
\hline
\end{tabular}


